\title{
DOCTORES IURIS DE LA REAL FÁBRICA DE TABACOS
}

ANTONIO MERCHÁN*

En principio este título puede sonar a tema o asunto dieciochesco como realmente es el caso de la sede o sitio que nos sirve de referencia. Pero ciertamente no es así; aunque sí se puede hablar de los Doctores iuris de la Real Fábrica de Tabacos hispalense. Ahora bien del presente siglo, porque en verdad todos los licenciados en derecho, a quienes se ha colacionado el grado de doctor en el siglo xx en y por la Universidad de Sevilla lo han defendido en la actual sede de la Facultad de Derecho Hispalense, la impresionante y simbólica Real Fábrica de Tabacos, que empezó a ser tal sede jurídica a mediados de los cincuenta.Y por desgracia para muchos, que sentimos el arraigo a un centro tan singular y tan nuestro -fuimos los pioneros de su ocupación universitaria casi cuando todavía era cuartel de artillería-, con la inminente entrada en el siglo XXI sufriremos por razones no suficientemente aclaradas una especie de destierro; y a partir de entonces ya no podrá hablarse más de Doctores iuris de la Real Fábrica de Tabacos, sino que habremos de referirnos a los Doctores iuris de la Pirotecnia -también antiguo cuartel militar-, donde las excavadoras rugen ya imparables preparando los cimientos de la incréble alternativa a nuestro indeseado desahucio.

Aprovechando esta acotación temporal, que dura casi medio siglo (1955-1999), realizada por razones de ubicación de la sede iuris de los estudios de doctorado, pretendemos exponer quiénes han sido los doctores en derecho durante la segunda mitad del siglo xx en la Facultad de Derecho de Sevilla y sobre todo cuáles fueron los temas que se seleccionaron y por tanto objeto de investigación para alcanzar el grado de doctor. Pues

* Universidad de Sevilla 
resulta que la acotación cronológica y espacial cobra también sentido científico, por cuanto que se trata de un espacio de tiempo en el que surge y se desarrolla la primera expresión de la autonomía científica de la Universidad de Sevilla moderna. En efecto, a mediados de los años cincuenta los estudios y la colación del grado de doctor se descentralizaron de la Universidad de Madrid y esta descentralización significó que las Universidades españolas y entre ellas la de Sevilla pudieran organizar esos estudios, nombrar el tribunal que juzga la tesis y otorgar dicho grado, quedando solamente a expensas de la administración central o Ministerio de Educación la expedición del título. Los estudios de doctorado constituyeron por tanto desde entonces, bastante antes que los de licenciatura, una expresión de particularismo científico de las universidades por mor de esa descentralización en su organización y colación; algo así como el preludio de lo que luego sería la autonomía universitaria proclamada en la Constitución y aplicada por la Ley de Reforma Universitaria de $1983 .{ }^{1}$

En este casi medio siglo de autonomía en los estudios de doctorado podemos distinguir, por tanto, dos etapas. Una primera que arranca, como hemos dicho, en el segundo lustro de los años cincuenta y llega hasta mediados de los ochenta, en la que se puede hablar de una autonomía de los estudios de doctorado fruto de la ruptura de la centralización en los estudios universitarios preconizada por la Ley de Ordenación Universitaria de 1943, provocada por el Real Decreto de 25 de junio de 1954 (BOE de 12 de julio) que permite y regula la colación del grado de doctor en todas las Universidades. Y otra segunda etapa a partir de 1985 (Real Decreto de 23 de enero de 1985, BOE de 16 de febrero, en el que se regula el tercer ciclo -nombre que ahora reciben los estudios de doctorado-) en la que se pone en práctica la autonomía universitaria sobre dichos estudios en virtud de la citada Ley de autonomía o reforma universitaria; etapa que por lo demás ha sido objeto de una cierta modificación en la estructuración de los estudios de doctorado con motivo del reciente Real Decreto de 30 de abril de 1998 (BOE de $1^{\circ}$ de mayo se 1998).

Estas matizaciones cronológicas, que cristalizan en esas dos etapas, tienen mucho sentido, como veremos más detalladamente. Pero de modo general nos interesa destacar ahora que la primera etapa, desde 1955 a 1985, de unos treinta años aproximadamente,

1. Acerca de la centralización del doctorado en la universidad liberal y la frustrada excepción a dicha centralización durante el Sexenio Revolucionario véase el interesante y documentado trabajo de Carlos Petit, "La Administración y el Doctorado: Centralidad de Madrid", Anuario de Historia del Derecho Español, LXVII, I (1997), 593-613, y especialmente los pintorescos doctorados sevillanos de Ángel Martínez Sousa en 1871 y de Antonio de la Iglesia y Peña en 1872 , en p. 608, nota 37 . Sobre el significado de la autonomía en la Ley de Reforma Universitaria de 1983 véase mi trabajo "Reforma y autonomía en la Universidad española tras la restauración democrática. El caso de la Universidad de Sevilla (1977-1992)", Università in Europa, Messina 1995, pp. 665-703. representa como es lógico la etapa más histórica. En efecto podemos estudiarla con más perspectiva y en muchos aspectos constituye un ciclo bastante completo de la evolución de los estudios de Doctorado en Derecho en la Universidad de Sevilla, como lo representa el hecho de que podamos seguir aspectos muy interesantes del currículum académico, político o social de los doctores. Por eso nuestra comunicación se va a centrar fundamentalmente en ella, sin que ello signifique que no dejemos de tocar aspectos generales que se refieren también a la segunda etapa (1986-2000).

En el fondo lo que pretendemos con nuestra comunicación es participar los primeros pasos de una investigación de mucha más envergadura que tendrá como contenido principal y general el estudio de la ciencia jurídica de la Universidad de Sevilla durante el siglo XX.

\section{UNA SIMPLE CUESTION DE NÚMEROS}

Los números totales: durante este casi medio siglo de doctorado en la Facultad de Derecho de Sevilla (1955-1999) se han defendido aproximadamente un total de casi trescientas tesis doctorales, concretamente a la altura de octubre de 1999 el número es de doscientas noventa. De ellas 137 corresponden a la primera etapa, es decir desde 1955 a 1985, etapa de 30 años anterior a la aplicación del doctorado regulado por la LRU; y un número de 153 durante la que consideramos la segunda etapa, esos quince años que van de 1985 a 1999, en la que se aplica la LRU. La comparación de estos datos de por sí es muy elocuente pues manifiesta cómo en los tres últimos lustros se han producido tantas o más tesis doctorales que en los tres primeros decenios. ¿Cuáles pueden ser las razones de este resultado tan prolífico? Tal vez presentando una secuencia por años podamos alumbrar algunas explicaciones.

Secuencia numérica por años. La primera tesis doctoral se lee en el año 1955, es decir inmediatamente después de la promulgación de la norma que permite los doctorados en las universidades de provincias. Curiosamente fue defendida por un doctor que alcanzó el orden episcopal y desempeñó su dignidad de Obispo en la diócesis de Cordoba durante los años setenta; se trata del Dr. José Antonio Infantes Florido. Al año siguiente, en 1956, se defiende también sólo una, pero en 1957 el número aumenta a cuatro y en 1958 a cinco, bajando a dos en 1959. Colacionaron el grado de doctor en este lustro de los años cincuenta en total doce doctores, lo que significa, por tanto, una media de producción doctoral de 2,2 tesis por año. Esta media asciende un punto en los años sesenta, concretamente a 3,3 tesis por año, aunque destacan el año 1965 en que 
se defienden cinco doctorados y el año 1969 en el que colacionan el grado de doctor siete doctorandos. En total se leyeron en esta década 33 tesis. En los años setenta casi se dobla el número de doctores, pues es de 64 ; lo que significa una media de 6,4 por año sobresaliendo los años 1972 con nueve tesis y once en el año 1976. El aumento de población universitaria determinó que la Ley General de Educación generara una reorganización universitaria que tuvo como expresiones la creación de nuevas universidades en las que nunca faltó la correspondiente Facultad de Derecho y en consecuencia la expectativa profesoral más amplia.

Esta tendencia ascendente se rompe en los años ochenta pues sólo llegan a leerse 46 tesis doctorales de las cuales veintisiete corresponden al lustro anterior a la iniciación de la aplicación de la LRU en materia de doctorado y diecinueve al lustro posterior. La media por tanto fue de 4,6 por año. Ello tal vez sea debido a la gran oferta de empleo que progresivamente deparó la organización y desarrollo político administrativo del Estado de las Autonomías programado en la Constitución de 1978. Ciertamente la nueva función pública en las Comunidades autónomas significó un atractivo y expeditivo modo de encontrar un puesto de trabajo funcionarial para quienes habían cursado la carrera de derecho y mermó dedicaciones a la enseñanza universitaria.

Todo lo contrario sucede en el decenio de los noventa, que es el que presenta mayor número de doctores, 133, número que se verá aumentado en dos o tres cuando acabe el siglo. Esto significa una media de casi catorce tesis por año y destacan considerablemente 1997 con 21 tesis y los años 94, 96 y 99 con 19 doctores. Ahora, al saturarse la oferta de la burocracia autonómica, el desarrollo de la Ley de Reforma Universitaria se erige en el determinante de una oferta de empleo inusitada para funcionarios docentes en las cuantiosas Facultades de Derecho y Económicas, Relaciones laborales y Escuelas de Graduados Sociales, etc., y para las que el doctorado constituye un factor curricular promocionante.

Sobre la secuencia numérica de las doctoras iuris, creemos que resultará interesante hacer algunas consideraciones numéricas, sobre la participación de la mujer en el doctorado. La primera mujer a la que se colacionó el grado de doctor en derecho por la Universidad de Sevilla fue la Dra. M ${ }^{a}$ Rosa Garrido Conde y tuvo lugar en 1965, concretamente en el área de Derecho del Trabajo; el tema de la tesis fue la intervención pública en la negociación colectiva en el Canadá y el director el jovencísimo y recién incorporado Catedrático de Derecho del Trabajo Dr. Miguel Rodríguez-Piñero. Desde entonces hasta hoy se han doctorado 68 mujeres; pero hay una gran diferencia entre la primera etapa, en la que alcanzaron el grado de doctor once, y la segunda cuyo número se quintuplicó, pues accedieron a ese grado cincuenta y siete mujeres. La participación femeni- na, por tanto, en el doctorado durante este medio siglo significa una cuota del 23,5 por ciento de las 290 tesis leídas hasta el día de hoy y está previsto el acceso de más doctoras antes del final de diciembre de 1999. En la etapa de la descentralización (1955-1985) la secuencia es muy baja y discontinua: una en 1965 como acabamos de ver; dos en 1972; una en 1974; una en 1978; una en 1980; dos en 1982; una en 1983; una en 1984; y una en 1985. Un total por tanto de once tesis defendidas por mujeres, lo cual significa menos del 10 por ciento de las 137 leídas.

En cuanto a la etapa de la aplicación de la LRU (1985-1999), si bien en el segundo lustro de los ochenta solamente defendieron la tesis doctoral dos mujeres, lo que equivale a una media de 0,4 , en el decenio de los noventa el número de doctoras ha aumentado de manera copiosa, como lo demuestra el hecho de una media de 5,7 tesis doctorales leídas; destacando el año 1997 en el que se confirió el grado de doctor a diez mujeres y lo alcanzaron ocho en 1999, no descartándose que este número aumente de aquí a final de siglo. Un total por tanto de 57 tesis leídas, lo que significa el 33 por ciento de las 153 leídas en esta etapa.

\section{CUESTIÓN DE TEMAS}

Consideramos este planteamiento el meollo de nuestra disertación: exponer los temas que se reflejan en las tesis doctorales leídas durante este medio siglo. Para ello vamos a realizar una primera aproximación temática que tiene como referencia las diferentes áreas de conocimiento, como unidades básicas, pues ya de por sí nos marcan una referencia temática sobre los grandes campos del saber jurídico. Con este motivo las vamos a integrar en grupos que están en función de unos segmentos numéricos, determinados por el número de tesis leídas. Esta operación la practicaremos de modo general a toda la época estudiada (1955-1999), y a cada una de las etapas en que la hemos dividido: de la descentralización (1955-1985) y de la autonomía (1985-1999). Luego practicaremos algunas reflexiones sobre ello. En todo caso dedicaremos un apartado especial a las doctoras iuris.

El siguiente paso estará representado por la exposición de los temas a los que se refieren las tesis doctorales defendidas en cada una de las áreas, con sus convenientes comentarios. En este punto en la presente comunicación sólo prestaremos exclusiva atención a las tesis doctorales de la primera etapa, que hemos denominado de la descentralización, es decir los treinta años de estudios de doctorado sevillano que contemplan desde 1955 a 1999 . 
Veamos en primer lugar la agrupación temática de las áreas de conocimiento en función del número de tesis leídas en cada una de ellas durante todo el período 1955-1999. Un primer grupo está representado por las áreas de conocimiento que superan el número total de treinta tesis. Este es el caso de Derecho Civil con 40; Derecho del Trabajo con 36; Derecho Penal con 34; y Derecho Administrativo con 32. Un segundo grupo lo constituyen las áreas de conocimiento que se sitúan en un número que ronda las veinte tesis doctorales. Esta es la circunstancia de Derecho Procesal con 23, Filosofia del Derecho con 21 tesis, Derecho Internacional con 20, y Derecho Mercantil con 18. Un tercer grupo lo integran las áreas en las que se promocionan entre diez y quince doctores. Este es el caso de Historia del Derecho con 15 tesis, Derecho Financiero, también con 15; y Derecho Político/Constitucional con 13. En un cuarto grupo se encuentran las áreas de Derecho Romano y de Derecho Canónico que no llegan a diez tesis doctorales cada una de ellas, concretamente ocho y cinco respectivamente; y además presentan en la segunda etapa una evolución descendente, tres en Romano y dos en Canónico.

Veamos ahora la agrupación temática de las áreas por el número de tesis leídas durante la primera etapa (1955-1985). Un primer grupo lo integran las áreas en las que se leyeron entre quince y veinte tesis: Derecho Administrativo con 20; Derecho del Trabajo con 20; y Derecho Civil con 17. Un segundo grupo reúne las áreas en las que se leyeron entre diez y quince tesis: Derecho Penal con 14; Historia del Derecho con 13; y Derecho Procesal con 10. En un tercer grupo se ubican las áreas en las que se defendieron entre diez y cinco tesis: Derecho Internacional con 8; Filosofía del Derecho con 7; Derecho Financiero y Derecho Político con 6; y Derecho Romano con 5. Un cuarto grupo que contiene a las áreas con menos de cinco tesis doctorales: Derecho Mercantil y Derecho Canónico, ambas con 3.Y por último pasemos a determinar la agrupación temática de las áreas durante la segunda etapa o de la autonomía de la LRU (1985-1999) en función del número de tesis leídas en cada una de ellas. Un primer grupo lo integran las áreas en las que se han defendido entre quince y veinticinco tesis doctorales: Derecho Civil con 23; Derecho Penal con 20; Derecho del Trabajo con 16. Un segundo grupo contempla las áreas en las que se han leído entre diez y quince tesis doctorales: Filosofia del Derecho y Derecho Mercantil con 15; Derecho Internacional y Derecho Administrativo con 12. Un tercer grupo de áreas que se integra por aquellas en las que se han presentado entre cinco y diez tesis doctorales: Derecho Financiero y Derecho Procesal con 9; y Derecho Político/Constitucional con 7. Un cuarto grupo de áreas que comprende aquellas en las que se han leído menos de cinco tesis doctorales: Derecho Romano con 3; Historia del Derecho y Derecho Canónico con 2.

Hagamos ahora algunas reflexiones comparativas sobre estas agrupaciones numéricas generales y por etapas, que tienen como referencia común las diferentes áreas de conocimiento o unidades temáticas, en definitiva, más sobresalientes.
Interesa destacar que así como la situación es claramente ascendente y prevalente durante la primera etapa para Derecho Administrativo y Derecho del Trabajo, esa tendencia se vuelve más o menos discretamente descendente durante la segunda etapa para dichas áreas; en tanto que continúan su evolución ascendente las áreas de Derecho Civil y de Derecho Penal, siendo aquella la que tiene un signo más claramente ascendente (de diecisiete a veintitrés). De otra parte la que más desciende en número de doctores es precisamente la que había alcanzado un número más alto en la primera etapa, es decir, Derecho Administrativo que baja de 20 a 12 tesis. Filosofia del Derecho tiene un signo ascendente muy pronunciado pues en la primera etapa se leen siete y en la segunda quince, la mayor parte de ellas dirigidas por el Catedrático de Filosofía del Derecho Antonio Enrique Pérez-Luño. Algo parecido podemos decir de Derecho Mercantil que de tres sube a quince, en gran medida determinado porque se rompe la tradición boloñesa y se prefiere el doctorado sevillano generalmente dirigido precisamente por antiguos doctores boloñeses, a saber los Catedráticos de Derecho Mercanti Profesores Olivencia Ruiz y su discípulo Jiménez Sánchez. Más discreto es el aumento de Derecho Internacional que pasa de ocho a doce tesis doctorales dirigidas en su mayoría por el Catedrático de Derecho Internacional Público Carrillo Salcedo. Por lo que se refiere al área de Derecho Procesal se observa un cierto mantenimiento del ritmo de la etapa anterior por cuanto simplemente desciende de diez a nueve. Llama la atención el enorme descenso de lecturas de tesis doctorales que se aprecia en la segunda etapa en el área de Historia del Derecho que baja a dos; la tónica ascendente de Financiero de seis a nueve, área también boloñesa como hemos advertido más arriba; y la permanencia o leve ascenso de Político/Constitucional de seis a siete.

Como fácilmente se puede observar, hay una considerable preferencia a favor de la tesis de las áreas jurídico-positivas; y dentro de éstas, en primer lugar por las de Derecho Civil, Derecho del Trabajo, Derecho Penal y Derecho Administrativo; en segundo lugar por las de Derecho Internacional, Derecho Procesal y Derecho Mercantil; y en tercero Derecho Financiero y Derecho Político/Constitucional. En cuanto a las jurídico-formativas destaca la de Filosofía del Derecho sobre todo en la segunda etapa con un signo claramente ascendente, lo cual contrasta con la evolución de la Historia del Derecho, que después de una primera etapa muy prolífica en la segunda apenas ha colacionado doctores. Las que menos doctores promocionan son las áreas de Derecho Romano y de Derecho Canónico. ${ }^{2}$

2. En todo caso, debemos hacer notar que estas reflexiones numéricas debẻn ser objeto de ciertas correcciones complementarias al alza, si se trata de cuantificar los doctores totales integrados o vinculados a la Facultad de Derecho durante las etapas que estudiamos. Pues en determinadas áreas, como son, sobre todo, las de Derecho Mercan- 
Si tenemos en cuenta la distribución por áreas de conocimiento de doctoras podemos decir que en la primera etapa del doctorado (1955-1985) las preferencias están muy repartidas: así encontramos dos tesis en Derecho del Trabajo, Derecho Penal y Derecho Romano; y una en Derecho Administrativo, Derecho Financiero y Derecho Civil, Historia del Derecho y Derecho Internacional. No hubo doctoras durante esta etapa en Derecho Canónico, Derecho Mercantil, Derecho Político, Derecho Procesal y Filosofía del Derecho. En la segunda etapa (1985-1999) destacan Derecho Civil y Derecho Penal con once doctoras; nueve en Derecho Internacional; siete en Derecho Procesal y seis en Derecho del Trabajo. De otra parte encontramos números más bajos, a saber: cinco en Derecho Administrativo; cuatro en Derecho Mercantil; dos en Derecho Constitucional/Político; y una en Derecho Romano y Filosofía del Derecho. En tanto que no hay doctoras durante esta segunda etapa en Derecho Financiero, Historia del Derecho y tampoco, como ya sabemos, en Derecho Canónico.

Como podemos ver al igual que sucede con el índice general de preferencias, en lo que acontece especialmente a las mujeres doctoras las áreas de derecho positivo también son las más solicitadas para realizar el doctorado; y dentro de ellas Derecho Civil, Penal y Derecho del Trabajo. Al contrario ocurre con las jurídico-formativas (Derecho Romano, Historia del Derecho y Filosofia del Derecho) en las que como hemos visto los números son muy bajos. Asimismo hay que hacer notar que en el área de Derecho Canónico no ha colacionado el grado de doctor ninguna mujer en la Facultad de Derecho de Sevilla hasta el momento.

\section{LOS TEMAS DOCTORALES QUE SON OBJETO DE INVESTIGACIÓN DURANTE LA ETAPA} DE LA DESCENTRALIZACIÓN (1955-1985)

Para presentar los temas que han sido objeto de investigación en cada área se me ocurre, en principio, que es adecuado realizarlo según el orden de prelación que otorga el hecho de la cuantía de tesis defendidas por área de conocimiento, que en gran medida se puede considerar indicativo de las preferencias por áreas que acabamos de exponer. Para ello seguiremos la sistemática de agrupamientos que hemos expuesto más arriba. Pasemos por tanto a tratar los temas doctorales durante la etapa de la descentralización (1955-1985)

iil, Derecho Financiero y Derecho del Trabajo, determinados doctorandos optaron por realizar y colacionar la tesis por la Universidad de Bolonia. También es verdad que un número considerable de ellos revalidaron su título de doctor -boloñés- colacionándolo a su vez por la Universidad de Sevilla.
1. Un primer grupo está constituido por Derecho Administrativo, Derecho del Trabajo y Derecho Civil, áreas en las que se han defendido entre quince y veinte tesis doctorales.

Derecho Administrativo. Salvo en contadas ocasiones las tesis doctorales de esta primera etapa fueron dirigidas por el Maestro de administrativistas sevillanos el Prof. Dr. Manuel Clavero Arévalo, Decano de la Facultad, Rector de la Universidad y Ministro del Gobierno de la Unión de Centro Democrático durante la Transición. Si atendemos a los temas destacan entre ellas cuantitativamente las que están dedicadas a los bienes públicos. Así las que se refieren: al concepto y naturaleza jurídica de los bienes comunales, con especial referencia a su reivindicación, de Jesús Aranda Navarro; ${ }^{3}$ al régimen jurídico administrativo de las playas de Lorenzo Martínez Escudero; ${ }^{4}$ al régimen jurídico administrativo de los caminos vecinales $y$ carreteras provinciales de Eduardo Baraja Carceller; ${ }^{5}$ al régimen jurídico-administrativo de las vías urbanas de Pedro Escribano Collado, actual Catedrático de Derecho Administrativo de la Facultad de Derecho de Sevilla, en la que ha desempeñado el cargo de Decano; ${ }^{6} \mathrm{o}$ al deslinde administrativo de Enrique Rivero Ysern que ejerce como Catedrático de Derecho Administrativo en la Universidad de Salamanca; ${ }^{7}$ o también a determinados bienes públicos o privados que por razones de salud pública están sometidos a una fuerte intervención administrativa como es el caso de las aguas minero-medicinales de Juan Antonio Cámpora Gamarra, que ha desempeñado la Secretaría del Ayuntamiento de Sevilla. ${ }^{8}$

Otros temas en los que se reincide aunque con menos frecuencia son los que tienen como objeto a la expropiación forzosa y a los funcionarios. Así encontramos las tesis doctorales que se refieren al justiprecio de Juan Antonio Tamayo Isasi-Isasmendi ${ }^{9} \mathrm{y}$ a la revisión en materia de expropiación forzosa de Alfonso Pérez Moreno, Catedrático de Derecho Administrativo en la Universidad de Sevilla y prestigiosísimo abogado administrativista; ${ }^{10} \mathrm{y}$ también en esta línea encontramos la tesis dedicada a la edificación forzosa y la

Jesús Aranda Navarro, Los bienes comunales: su concepto y naturaleza juridica; especial referencia al problema de su reivindicación, 1962; Director: Manuel Clavero Arévalo; FDEB TD-023.

4. Lorenzo Martínez Escudero, El régimen jurídico administrativo de las playas, 1969; Director: Manuel Clavero Aréalo; FDEB TD 043

Eduardo Baraja Carceller, Caminos vecinales $y$ carreteras provinciales, 1972; Director: Manuel Clavero Arévalo; FDEB TD-058.

6. Pedro Escribano Collado, Régimen jurídico-administrativo de las vías urbanas, 1973; Director: Manuel Clavero Arévalo; FDEB TD-068,

7. Enrique Rivero Ysern, El deslinde administrativo, 1966; Director: Manuel Clavero Arévalo; FDEB TD-032. 8. Juan Antonio Cámpora Gamarra, Régimen juŕdico de las aguas minero medicinales, 1964; Director: Manuel Clavero Arévalo; FDEB TD-025.

9. Juan Antonio Tamayo Isasi-Isasmendi, El justiprecio en la expropiación forzosa, 1964; Director: Manuel Clavero Arévalo; FDEB TD-026,

10. Alfonso Pérez Moreno, La reversión en materia de expropiación forzosa, 1967; Director: Manuel Clavero Arévalo FDEB TD-034 
especulación del suelo de Federico Romero Hernández. ${ }^{11} \mathrm{Y}$ por lo que acontece al tema de los funcionarios encontramos dos tesis, una con un planteamiento más general sobre las situaciones administrativas de los funcionarios en la Administración Civil del Estado de José Torreblanca Vergara, del Cuerpo Técnico de la Administración Civil del Estado, que durante muchos años ha ejercido como tal en el Rectorado de la Universidad de Sevilla; ${ }^{12}$ y otra de asunto más particular sobre el problema de las incompatibilidades de José Muñoz González. ${ }^{13}$ Acerca del tema de la actividad administrativa de fomento encontramos un estudio sobre el fomento a la industria privada de José Ignacio López González, que actualmente ejerce la docencia como Catedrático de la Universidad Pablo de Olavide; ${ }^{14}$ en cuanto a los servicios públicos se ha estudiado el de abastecimientos de aguas a las poblaciones de José $\mathrm{M}^{\mathrm{a}}$ Garrido Lopera; ${ }^{15} \mathrm{y}$ por lo que se refiere a los contratos administrativos ha sido objeto de investigación el contrato de suministro de José Luis Rivero Ysern, Catedrático de Universidad de la Facultad de Derecho de Sevilla y actual Vicedecano de la misma. ${ }^{16}$

Otros temas se provectan sobre determinados aspectos de las diferentes administraciones. Así a la administración territorial la contempla el estudio sobre la historia y actualidad de la región de Amparo Rubiales Torrejón, segunda mujer doctora por la Universidad de Sevilla, Profesora Titular de Derecho Administrativo, que ha jugado un relevante y comprometido papel sociopolítico durante la Transición Política, como Concejal del Ayuntamiento sevillano, Diputada del Parlamento Autonómico y del Nacional, Senadora y Delegada del Gobierno en Andalucía. ${ }^{17} \mathrm{~A}$ la administración municipal se refieren dos tesis doctorales; una sobre el alcalde en la organización administrativa española de José Ignacio Morillo Velarde Pérez, actualmente Catedrático de Derecho Administrativo en la Universidad de Cádiz; ${ }^{18}$ y otra sobre la población municipal de Antonio Bullón

1. Federico Romero Hernández, La edificación forzosa y la especulación del suelo, 1969; Director: Manuel Clavero Arévalo; FDEB TD-042.

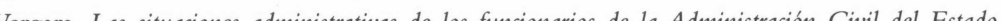
1977; Director: Manuel Clavero Arévalo; FDEB TD-101. 3. José Muñoz González, El problema de las incompatibilidades en España, 1981; Director: Alfonso Pérez Moreno

4. José Ignacio López González, Estudio de la actividad administrativa de fomento a la industria privada, 1972; DirecJorero Arévalo; FDEB TD-063.

5. José María Garrido Lopera, El servicio público de abastecimiento de agua a poblaciones, 1972; Director: Manuel FDEB TD-057.

José Luis Rivero Ysern, El contrato administrativo de suministro, 1975; Director: Manuel Clavero Arévalo; FDEB TD-082.

7. Amparo Rubiales Torrejón, El espacio regional: historia y actualidad, 1972; Director: Manuel Clavero Arévalo; FDEB TD-064.

18. José Ignacio Morillo Velarde Pérez, El alcalde en la organización administrativa española, 1976; Director: Manuel Clavero Arévalo; FDEB TD-088.
Ramírez. ${ }^{19}$ A la administración financiera se dedica la investigación sobre la historia y ámbito de lo económico-administrativo de Pedro Luis Serrera Contreras, Profesor asociado y actual Decano del Cuerpo de Abogados del Estado de Sevilla. ${ }^{20} \mathrm{Y}$ en cuanto a la administración militar encontramos la tesis sobre la ordenación jurídica de la defensa nacional de Federico Sánchez de Velasco, Profesor asociado y General auditor del Ejército de Tierra. ${ }^{21}$ Finalmente, en esta primera etapa encontramos un estudio sobre las fuentes del derecho administrativo que se centra en la investigación del principio de irretroactividad en las normas jurídico-administrativas de Francisco López Menudo, que ejerce actualmente como Catedrático de Derecho Administrativo de la Universidad de Sevilla y es vocal del Consejo Consultivo Andaluz. ${ }^{22}$

Derecho del Trabajo. Las tesis correspondientes a este área de conocimiento durante esta primera etapa contemplan aspectos muy diversos de la misma. Un grupo de ellas están referidas a asuntos relativos a los elementos del contrato de trabajo, así: la renuncia de los derechos del trabajador que la relación de trabajo comporta de Antonio Ojeda Avilés, quien ejerce como Catedrático de Universidad en la Facultad de Derecho de Sevilla; ${ }^{2 .}$ las modificaciones de la prestación de trabajo de Jesús Cruz Villalón, Catedrático de Derecho del Trabajo en la Universidad de Cádiz. ${ }^{24}$ El salario ha sido estudiado en su modalidad de a rendimiento por José Rodríguez de la Borbolla Camoyán, Profesor Titular de Derecho del Trabajo y Ex-Presidente de la Junta de Andalucía; ${ }^{25}$ y la prohibición de competencia y el contrato de trabajo por Jaime Castiñeira Fernández, Profesor Titular de Derecho del Trabajo en la Universidad de Sevilla. ${ }^{26}$ Otras tesis doctorales están referidas a relaciones de trabajo que presentan especiales peculiaridades o son impropias por razón del sujeto que lo presta, como la relación laboral de los empleados públicos de Miguel Rodríguez-Piñero Bravo-Ferrer, sucesor del Profesor Alonso Olea en la Cátedra de Derecho

19. Antonio Bullón Ramírez, La población municipal: evolución histórica de su clasificación. Análisis y efectos de la misma en la legislación vigente, 1958; Director: Manuel Clavero Arévalo; FDEB TD-007.

20. Pedro Luis Serrera Contreras, Lo económico-administrativo historia y ámbito, 1965; Director: Manuel Clavero Arévalo / Jaime García Añoveros; FDEB TD-029.

21. Federico Sánchez de Velasco, La ordenación jurídica de la defensa nacional, 1981; Director: Alfonso Pérez Moreno; FDEB TD-118.

22. Francisco López Menudo, El principio de irretroactividad en las normas jurídico-administrativas, 1981; Director Alfonso Pérez Moreno; FDEB TD-116.

23. Antonio Ojeda Avilés, La renuncia de derechos del trabajador, 1970; Director: Miguel Rodríguez Piñero Bravo Ferrer; FDEB TD-047.

24. Jesús Cruz Villalón, Las modificaciones de la prestación de trabajo, 1982; Director: Miguel Rodríguez Piñero; FDEB TD-123.

25. José Rodríguez de la Borbolla Camoyán, El salario a rendimiento, 1974; Director: Miguel Rodríguez-Piñero Bravo-Ferrer; FDEB TD-078.

26. Jaime Castiñeira Fernández, Prohibición de competencia $y$ contrato de trabajo, 1976; Director: Miguel Rodríguez Piñero Bravo Ferrer; FDEB TD-096. 
del Trabajo sevillana y junto con éste promotor de los doctorados que se leen durante esta etapa. A mayor abundamiento de la proyección jurídico social del Dr. RodríguezPiñero debemos decir que ha desempeñado los cargos de Decano de la Facultad de Derecho hispalense, Magistrado del Tribunal Constitucional y Presidente del mismo; y que actualmente es miembro del Consejo de Estado. ${ }^{27}$ En esta línea de contratos de trabajo especiales encontramos también el estudio de la relación contractual de los futbolistas profesionales de José Cabrera Bazán, Catedrático de Derecho del Trabajo de la Universidad de Cádiz y Senador durante varias legislaturas; ${ }^{28}$ y la tesis sobre la relación laboral que presta el personal de las confederaciones hidrográficas del prestigioso abogado administrativista sevillano Antonio Pérez Marín; ${ }^{29}$ o la que tiene como sujetos laborales a los emigrantes de José Serrano Carvajal. ${ }^{30}$ Acerca de la dirección y disciplina en la empresa encontramos dos estudios; uno sobre el poder de dirección del empresario de Alfredo Montoya Melgar, Catedrático de Derecho del Trabajo de la Universidad de Murcia; ${ }^{31} \mathrm{y}$ otro sobre la responsabilidad disciplinaria laboral del trabajador de Bernardo $\mathrm{M}^{\mathrm{a}}$ Cremades, Catedrático de Derecho del Trabajo, promotor de la institución del arbitraje y miembro de las Cortes de Arbitraje más prestigiosas del mundo. ${ }^{32}$

A la negociación colectiva y a los conflictos que comporta están dedicadas las tesis doctorales que tienen como objeto los temas siguientes: la representación colectiva en la empresa de Joaquín Cuevas López; ${ }^{33}$ la negociación colectiva y la paz laboral de Salvador del Rey Guanter, Catedrático de Derecho del Trabajo de la Universidad Pompeu Fabra; ${ }^{34}$ la intervención pública en la negociación colectiva en Canadá de Rosa María Garrido Conde, que fue la primera mujer doctora de la Facultad de Derecho de Sevilla; ${ }^{35}$ y la intervención del Estado en los conflictos colectivos de trabajo de Fermín Rodríguez-Sañudo, Cate-

27. Miguel Rodríguez-Piñero Bravo-Ferrer, La relación laboral de los empleados públicos, 1959; Director: Manuel Alonso Olea; FDEB TD-013.

28. José Cabrera Bazán, La relación contractual de los futbolistas profesionales, 1960; Manuel Alonso Olea; FDEB TD-016.

29. Antoro Pérez Marín, En torno al personal de las Confederaciones Hidrográficas, 1974: Director: Miguel Rodríguez-Piñero Bravo-Ferrer; FDEB TD-077.

30. José Serrano Carvajel La emigución español y su régimen jurídico, 1965; Director: Manuel Alonso Olea. FDEB 30. José

TD-031. TD-021.

32. Bernardo María Cremades, La responsabilidad disciplinario-laboral del trabajador, 1968; Director: Miguel Rodríguez Piñero; FDEB TD-037.

33. Joaquín Cuevas López, Estructura y función de la representación colectiva en la empresa, 1980; Director: Miguel Rodríguez Piñero Bravo Ferrer; FDEB TD-156.

34. Salvador del Rey Guanter, Negociación colectiva y paz laboral, 1982; Director: Miguel Rodríguez-Piñero BravoFerrer; FDEB TD-126.

35. Rosa María Garrido Conde, La intervención pública en la negociación colectiva en el Canadá, 1965; Director: Miguel Rodríguez Piñero Bravo Ferrer; FDEB TD-030. drático de Derecho del Trabajo de la Universidad Pablo de Olavide ${ }^{36}$ También encontramos tesis doctorales sobre derecho sindical: así el estudio histórico-jurídico sobre la asociación obrera de Manuel Ramón Alarcón Caracuel, Catedrático de Derecho del Trabajo y actual Decano de la Facultad de Derecho hispalense; ${ }^{37}$ otro sobre la estructura y naturaleza de la asociación sindical de María Fernanda Fernández López, Catedrática de Derecho del Trabajo de la Universidad de Sevilla; ${ }^{38}$ y uno tercero más concreto sobre la conciliación sindical de Alfredo Torres Orozco. ${ }^{39}$ Asimismo se han estudiado aspectos de la seguridad en el trabajo, como los que hacen referencia al accidente de trabajo tanto in itinere de José $\mathrm{M}^{\mathrm{a}}$ Vallejo Muguruza; ${ }^{40}$ como por acto de tercero de Manuel Chaves González, Profesor Titular de Derecho del Trabajo, ex-Ministro de Trabajo y actual Presidente de la Junta de Andalucía. ${ }^{41}$ Finalmente debemos citar determinadas tesis doctorales que tienen como temas aspectos de la relación laboral que persiguen garantizar el salario del trabajador: así el que estudia la protección legal de los trabajadores en los supuestos de insolvencia del empleador de Manuel Pérez Pérez, Profesor Titular de Derecho del Trabajo en la Facultad sevillana, ${ }^{42}$ y el que está dedicado al fondo de garantía salarial de Joaquín García Murcia, que ejerce de Catedrático de Derecho del Trabajo en la Universidad hispalense. ${ }^{43}$

Derecho Civil. En esta primera etapa encontramos trabajos doctorales referidos a los más diversos grandes apartados del Derecho Civil. Así por lo que se refiere a lo que se considera la parte general encontramos dos estudios dedicados al derecho de la persona: uno debido a José L. García Hirschfeld acerca de las cuestiones prejudiciales del estado civil de las personas; ${ }^{44}$ y otro de Ángel López López, Catedrático de Derecho Civil de la Universidad hispalense, ex-Consejero de Gobernación de la Junta de Andalucía y ExPresidente del Parlamento Andaluz, sobre la posesión del estado familiar. ${ }^{45}$ Asimismo

36. Fermín Rodríguez-Sañudo Gutiérrez, La intervención del Estado en los conflictos colectivos de trabajo, 1971; Director: Miguel Rodríguez-Piñero / Manuel Alonso Olea; FDEB TD-056.

37. Manuel Ramón Alarcón Caracuel, La asociación obrera en el derecho histórico español (1839-1900), 1973. Director: Antonio Martín Valverde; FDEB TD-070

38. María Fernanda Fernández López, La asociación sindical: su estructura y naturaleza, 1982; Director: Miguel Rodríguez Piñero Bravo Ferrer; FDEB TD-120.

39. Alfredo Torres Orozco, La conciliación sindical en el derecho del trabajo español, 1961; Director: Manuel Alonso Olea; FDEB TD-019.

40. José $\mathrm{M}^{2}$ Vallejo Muguruza, Accidentes in itinere, 1959; Director: Manuel Alonso Olea; FDEB TD-12. 41. Manuel María Chaves González, La responsabilidad por acto de tercero en el accidente de trabajo, 1973; Director: Miguel Rodríguez Piñero Bravo Ferrer; FDEB TD-071.

42. Manuel Pérez Pérez, La protección legal de los trabajadores en los supuestos de insolvencia del empleador, 1979; Director: Miguel Rodríguez Piñero Bravo Ferrer; FDEB TD-106.

43. Joaquin Garcia Murcia, El fondo de garantia salarial, 1982; Director: Antonio Martín Valverde; FDEB TD-124. 44. José Luis García Hirschfeld, Cuestiones prejudiciales sobre el estado civil de las personas, 23.VI. 1972; Director: Juan B. Jordano Barea; FDEB TD-060.

45. Ángel Manuel López López, La posesión de estado familiar, 1970; Director: Juan Jordano Barea; FDEB TD-048. 
dentro de este gran apartado podemos incluir la tesis doctoral de Antonio Gordillo Cañas, Catedrático de Derecho Civil de la Facultad sevillana, sobre la representación aparente, perfilada como una aplicación singular del principio general de la protección de la apariencia. ${ }^{46}$

En el campo de los derechos reales hallamos el estudio de Antonio Gullón Ballesteros, Catedrático de Derecho Civil en la Universidad Complutense, sobre el derecho real de hipoteca. ${ }^{47} \mathrm{Y}$ en lo que acontece al derecho de obligaciones: el estudio de la cláusula resolutoria tácita del artículo 1.124 realizado por José Luis Fernández Cantos; ${ }^{48}$ la tesis sobre la acción subrogatoria de Juan Gómez Calero; ${ }^{49}$ y la responsabilidad por actos dañosos de la administración pública sometidos al derecho privado de Charles Louis Verhoeven. ${ }^{50}$

Por lo que acontece al derecho de contratos nos topamos con la investigación doctoral de Antonio Molina García sobre la doble venta a través de la jurisprudencia; ${ }^{51}$ la tesis doctoral de Francisco Capilla Roncero, Catedrático de Derecho Civil de la Universidad hispalense, sobre la estructura patrimonial y la personalidad jurídica de la sociedad civil, ${ }^{52}$ el estudio de José Codes Anguita acerca de la ley de 23 de julio de 1908 (Ley Azcárate) sobre la nulidad de los préstamos usurarios y su tratamiento en la Jurisprudencia del Tribunal Supremo; ${ }^{53}$ el contrato atípico de arrendamiento de automóviles de Javier Moreno Álvarez, Profesor Titular de Universidad de la Facultad sevillana; ${ }^{54}$ el contrato ganadero de reposición de José Antonio Infantes Florido, primer Doctor en Derecho por la Universidad de Sevilla, a partir de la descentralización del doctorado, y que desempeñó el obispado de la Diócesis de Córdoba durante los años setenta; ${ }^{55}$ y las mejoras fundiarias en el contrato de arrendamientos rústicos de Manuel Álvarez Fuentes. ${ }^{56}$

46. Antonio Gordillo, La representación aparente: una aplicación singular del principio general de protección de apariencia, 1977; Director: Juan B. Jordano Barea; FDEB TD-099.

47. Antonio Gullón Ballesteros, El derecho real de subhipoteca, 1966; Director: Alfonso Cossío y Corral; FDEB TD-002.

48. José Luis Fernández Cantos, El artículo 1124 del código civil español (la cláusula resolutoria tácita), 1957; Director: Afonso de COs Corral: FDEB TD-005.

9. Juan Gómez Calero, La acción subrogativa, 1958; Director: Alfonso de Cossío y Corral; FDEB TD-008.

50. Charles Louis Verhoeven, La responsabilidad por actos dañosos de la administración pública sometidos al derecho privado, 1983; Director: Ángel María López y López; FDEB TD-130

51. Antonio Molina García, La doble venta a través de la jurisprudencia, 1974; Director: Juan B. Jordano Barea; FDEB TD-080.

52. Francisco Capilla Roncero, Estructura patrimonial y personalidad juríica de la sociedad civil, 1981; Director: Ángel María López y López; FDEB TD-117.

53. José Luis Codes Anguita, La Ley del 23 de julio de 1908 sobre nulidad de préstamos usurarios y su tratamiento en la jurisprudencia del Tribunal Supremo, 1983; Director: Ángel María López y López; FDEB TD-129.

54. Javier Moreno Álvarez, El contrato atípico de arrendamiento de automóviles, 1972; Director: Juan B. Jordano Barea; FDEB TD-061.

55. José Antonio Infantes Florido, El contrato ganadero de reposición: Su naturaleza jurídica, 1955; Director: Alfonso de Cossio y Corral; FDEB TD-001

56. Manuel Álvarez Fuentes, Las mejoras fundiarias, 1969; Director: Juan Jordano Barea; FDEB TD-44.
Dentro del campo del derecho de familia podemos incluir el estudio doctoral de fuerte contenido histórico-jurídico sobre el régimen económico matrimonial regulado por el denominado Fuero del Baylio, ${ }^{57}$ y asimismo el estudio sobre los pactos de separación de hecho en el derecho común a partir de la ley de 2 de mayo de 1975 de Rosario Valpuesta, primera mujer Catedrática de la Facultad de Derecho de Sevilla y actualmente Rectora de la Universidad Pablo de Olavide ${ }^{58} \mathrm{Y}$ por lo que se refiere a la relación paterno filial la tesis doctoral sobre la adopción en el derecho comparado de Ángel Pizarro Rodríguez. ${ }^{59}$ En cuanto al derecho de sucesiones hemos detectado la tesis doctoral de Manuel de Cossío Martínez, Profesor Titular de Derecho Civil, sobre la sustitución fideicomisaria ${ }^{60}$ y la de José León-Castro Alonso, Catedrático de Derecho Civil, sobre la reserva ordinaria; ${ }^{61}$ ambos docentes de la Facultad de Derecho hispalense.

2. Un segundo grupo está referido a las áreas en que se han leído entre diez y quince tesis doctorales, en Derecho Penal, Historia del Derecho y Derecho Procesal.

Derecho Penal. La mayoría de las tesis doctorales fueron dirigidas durante esta etapa por el Catedrático de Derecho Penal Dr. José $M^{a}$ Navarrete Urieta, ya fallecido, y suelen ser trabajos de investigación que están dedicados al estudio de determinados delitos. De esta índole encontramos los estudios siguientes: el delito de alzamiento de bienes de Francisco Muñoz Conde, discípulo de aquél y como veremos sobre todo en la segunda etapa, prolífico director de tesis doctorales; hasta hace poco Catedrático de Derecho Penal en la Universidad de Sevilla y actualmente lo es en la Pablo de Olavide, recientemente ha sido distinguido con el Premio Humboldt de Ciencias Jurídicas; ${ }^{62}$ el delito de apropiación indebida de José Antonio Sainz-Pardo, Profesor Titular de Derecho Penal y actualmente Viceconsejero de Gobernación y Justicia del Gobierno Autónomo de Andalucía; ${ }^{63}$ el de receptación de Juan Antonio Martos Núñez, Profesor Titular de Dere-

57. Manuel Madrid del Cacho, El Fuero del Baylio. Estudio histórico-jurídico de la institución, 1961; Director: Alfonso de Cossío y Corral; FDEB TD-018.

58. María del Rosario Valpuesta Fernández, Los pactos de separación de hecho en derecho común tras la Ley de 2 de mayo de 1975, 1980; Director: Ángel López y López; FDEB TD-111.

59. Ángel Pizarro Rodrí Anez, La adopión: derecho comparado 1963. Director: Alfonso de Cossío y Corral: FDEB 59. Ange

60. Mar de Cossí Martínez En tormo a la sustitución fideicomicaria, 1970: Director: Juan B. Jordano Barea FDEB TD-049.

61. José Ricardo León-Castro Alonso, La reserva ordinaria, 1973; Director: Alfonso de Cossío y Corral; FDEB TD-073.

62. Francisco Muñoz Conde, El delito de alzamiento de bienes, 1970; Director: José $\mathrm{M}^{2}$ Navarrete Urieta; FDEB TD-050.

63. José Antonio Sainz-Pardo Casanova, El delito de apropiación indebida, 1973; Director: José $\mathrm{M}^{2}$ Navarrete Urieta; FDEB TD-072. 
cho Penal en la Facultad de Derecho de Sevilla; ${ }^{64}$ el de falsedad en documento privado de Enrique Casas Barquero, Profesor Titular de Derecho Penal de la Facultad de de Derecho de Córdoba, recientemente fallecido; ${ }^{65}$ y el de deserción militar de Antonio Millán Garrido; ${ }^{66} \mathrm{o}$ el dedicado a las faltas penales $y$ administrativas contenidas en el Código penal vigente en 1979 de Aníbal Ollero Sierra, Profesor Asociado de Derecho Penal perteneciente al Cuerpo Judicial. ${ }^{67}$

Aparte de un estudio historiográfico dedicado al penalista del siglo XIX Joaquín Francisco Pacheco realizado por Francisco Candil, Profesor Titular de Derecho Penal en la Universidad de Sevilla, ${ }^{68}$ en lo que se refiere a la parte general de la asignatura encontramos estudios como el dedicado a los elementos subjetivos del injusto en el Código penal español de Miguel Polaino, Catedrático de Derecho Penal en la Universidad de Sevilla, también discípulo de Navarrete Urieta y que ha dirigido un número importante de tesis doctorales; ${ }^{69}$ la imputabilidad y el alcoholismo de Andrés Posada de Pazos; ${ }^{70}$ la conspiración para cometer un delito de Joaquín Cuello Contreras, Catedrático de Derecho Penal de la Universidad de Extremadura y Magistrado del Tribunal Superior de Justicia de dicha Comunidad Autónoma; ${ }^{71}$ o los antecedentes penales de Manuel Grosso Galván, Profesor Titular de Derecho Penal en la Facultad de Derecho de Sevilla. ${ }^{72}$ Otros tienen un sentido más sociológico, como los factores asociales de la contracultura de José Luis García Prieto; ${ }^{73}$ o la delincuencia femenina en la provincia de Sevilla de María Victoria Agudo López. ${ }^{74} \mathrm{Y}$ por lo que se refiere a los cuerpos de seguridad encontramos un estudio sobre el cuerpo de policía local en la realidad policial española de Torcuato Pérez de Guz-

64. Juan Antonio Martos Núñez, El delito de receptación, 1983; Director: José María Navarrete Urieta; FDEB TD-127.

65. Enrique Casas Barquero, El delito de falsedad en documento privado, 1982; Director: Miguel Polaino Navarrete; FDEB TD-125.

66. Antonio Millán Garrido, El delito de deserción militar, 1981; Director: Miguel Polaino Navarrete; FDEB TD-115.

67. Aníbal Ollero de Sierra, Las faltas penales y administrativas contenidas en el código penal, 1979; Director: Josê María Navarrete Urieta; FDEB TD-107.

68. Francisco Candil Jiménez, D. Joaquín Francisco Pacheco: penalista del siglo XIX, 1973; Director: José María Navarrete Urieta; FDEB TD-069.

69. Miguel Polaino Navarrete, Los elementos subjetivos del injusto en el Código penal español, 1971; Director: José María Navarrete Urieta; FDEB TD-052.

70. Andrés Posada de Pazos, Imputabilidad y alcoholismo, 1967; Director: José $M^{2}$ Navarrete Urieta; FDEB TD-035. 71. Joaquín Cuello Contreras, La conspiración para cometer el delito: interpretación del art. 4,I CP, 1976; Director: José María Navarrete Urieta; FDEB TD-092.

72. Manuel Grosso Galván, Los antecedentes penales, 1982; Director: Francisco Muñoz Conde; FDEB TD-121; publicada en Barcelona, Bosch, 1983.

73. José Luis García Prieto, Factores asociales de la contracultura, 1978; Director: José María Navarrete Urieta; FDEB TD-102.

74. María Victoria Agudo López, La delincuencia femenina de Sevilla: aspectos fenomenológicos y etiologicos, 1974; Director: José María Navarrete Urieta; FDEB TD-081 mán Moore, perteneciente al Cuerpo de Jueces y Magistrados con destino en la Audiencia Provincial de Sevilla. ${ }^{75}$

Historia del Derecho. En este área de conocimiento llama la atención la importante producción de tesis doctorales que tiene lugar a partir del segundo lustro de los año sesenta, cuando se incorpora como Catedrático-Director de la misma el recientemente fallecido Prof. Dr. José Martínez Gijón. Bajo su dirección se realizó un elenco de trabajos científicos para acceder al grado de doctor que tienen de común el haber sido todos editados, obtener un número importante de ellos el Premio Extraordinario del Doctorado y el hecho de que casi todos sus autores hayan accedido a la Cátedra universitaria.

Los temas tratados se refieren tanto al derecho público-administrativo como al derecho privado, aunque son estos últimos los que predominan, con un fuerte reflejo de la especialidad mercantil tan querida y trabajada científicamente por el inolvidable Maestro de historiadores del derecho. Así, por lo que se refiere a la Historia de la Administración encontramos el estudio sobre la teoría general del oficio público de José García Marín, actualmente Catedrático en la Universidad Pablo de Olavide, estudio que ya ha alcanzado la segunda edición; ${ }^{76}$ o investigaciones más concretadas institucionalmente como son las que se refieren a la administración territorial indiana sobre las Presidencias-Gobernaciones en Indias de Fernando Muro Romero, Profesor Titular de Derecho Indiano en la Universidad de Sevilla; ${ }^{77}$ o sobre el cacique en el Virreinato del Perú de quien fuera prestigiosísimo historiador del Derecho Indiano y Catedrático de la Universidad de Huelva recientemente fallecido Carlos Díaz Rementería. ${ }^{78}$ Las hay también que tienen como objeto el régimen municipal, tanto en la Edad Media, cual es el caso del estudio sobre los factores de diferenciación social en los Fueros municipales del actual Catedrático de Historia del Derecho en la Universidad de Cádiz Alberto García Ulecia; ${ }^{79}$ o también el trabajo de investigación de ubicación temporal más presentista referido a la incidencia de los principios de centralización y descentralización en el régimen municipal español del siglo XIX de José Manuel Cárdenas Rodríguez de Moya. ${ }^{80}$

75. Torcuato Pérez de Guzmán Moore, Los cuerpos de policía local: el caso español, 1975; Director: José Jiménez 75. Torcuato Pêrez de
Blanco; FDEB TD-083.

76. José María García Marín. Teoría general del oficio público en Castilla durante la Baja Edad Media, 1972; Director 76. José María García Marín, Teoría

José Martínez Gijón; FDEB TD-066.
77. Fernando Muro Romero, Las Presidencias-gobernaciones en Indias: durante el siglo XVI, 1973; Director: José Mar77. Fernando Muro Romero,

78. Carlos J. Díaz Rementería, El cacique en el virreinato del Perú: estudio historico-jurídico, 1976; Director; José Martí78. Carlos J. Díaz Rement

79. Alberto García Ulecia, Factores de diferenciación jurídico-social en los fueros municipales de la Extremadura castellanoaragonesa, 1974; Director: José Martínez Gijón; FDEB TD-079.

80. José Manuel de Cárdenas y Rodríguez de Moya, La incidencia de los principios de centralización y descentralización en el régimen municipal español (1808-1874), 1979; Director: José Martínez Gijón; FDEB TD-108. 
En cuanto a la historia del derecho privado, encontramos estudios doctorales sobre la propiedad feudal, cual es el caso del mayorazgo del sumamente prolífico doctor Bartolomé Clavero, Catedrático de Historia del Derecho en la Universidad de Sevilla, de enorme prestigio internacional y cuya tesis ha sido objeto de varias ediciones; ${ }^{81} \mathrm{o}$ de derecho de familia, cuales son el referido a la filiación ilegítima de Enrique Gacto, Catedrático de Historia del Derecho de la Universidad de Murcia, ${ }^{82}$ o el que tiene como objeto de investigación la tutela de los huérfanos de Antonio Merchán, Catedrático de Historia del Derecho de la Facultad de Derecho hispalense. ${ }^{83} \mathrm{Y}$ por lo que respecta a la historia del derecho mercantil, aparte del estudio sobre la quiebra de Juan Antonio Alejandre, Catedrático de Historia del Derecho de la Universidad Complutense, ${ }^{84}$ continuando la línea investigadora del Maestro Martínez Gijón se realizaron dos sobre compañías. Uno referido a las reales compañias de comercio con América de Raquel Rico, Profesora Titular de Historia del Derecho en la Universidad de Sevilla, ${ }^{85}$ y otro a las compañias mercantiles en Bilbao realizado por Carlos Petit, Catedrático durante varios años en la Universidad Autónoma de Barcelona y actualmente en la Universidad de Huelva. ${ }^{86}$ De otra parte hay que señalar cómo en la Facultad de Derecho se defendieron tesis de Historia del Derecho Indiano -asignatura incardinada a la Facultad de Geografia e Historia- que tuvieron como objeto el juicio de residencia de José Luis Campuzano Zamalloa ${ }^{87}$ y las capitulaciones indianas de Rafael Diego Fernández-Sotelo. ${ }^{88}$

Derecho Procesal. Todas las tesis doctorales leídas en esta primera etapa fueron dirigidas por el Catedrático de Derecho Procesal don Faustino Gutiérrez-Alviz Armario. La mayoría de ellas tratan sobre determinados aspectos del proceso civil. Unos son más concretos como el allanamiento a la demanda de Jerónimo Infantes Florido; ${ }^{89}$ la prueba de

1. Bartolomé Clavero, Historia institucional del mayorazgo castellano, 1972; Director: José Martínez Gijón; FDEB TD-067

2. Enrique Gacto Fernández, La filiación natural en el derecho histórico español, 1968; Director: José Martínez Gijón; FDEB TD-039.

3. Antonio Merchán Alvarez, La tutela de los huérfanos en el derecho castellano-leonés desde la época hispano-romana hasta fines del siglo XV, 1975; Director: José Martínez Gijón; FDEB TD-086.

84. Juan Antonio Alejandre García, La quiebra en el derecho histórico español anterior a la codificación, 1969; Director: osé Martínez Gijón; FDEB TD-040.

5. Raquel Rico Linaje, El ejercicio del poder social en las Reales Compañias de Comercio con America, 1978; Director: Jsé Martínez Gijón; FDEB TD-104.

6. Carlos Petit, Compañias mercantiles en Bilbao (1737-1829), 1979; Director: José Martínez Gijón; FDEB TD-110. 87. José Luis Campuzano Zamalloa, La residencia del licenciado Rodrigo de Figueroa, 1957; Director: Guillermo Céspedes del Castillo; FDEB TD-006.

8. Rafael Diego Fernández-Sotelo, Régimen jurídico de las capitulaciones indianas (1492-1506), 1985; Director: Antonio Muro Orejón; FDEB TD-134.

99. Jerónimo Infantes Florido, El allanamiento a la demanda en el proceso civil español, 1957; Director: Faustino utiérrez-Alviz Armario; FDEB TD-004 informes de José Almagro Nosete, Catedrático de Derecho Procesal en la Universidad Complutense; ${ }^{90}$ las diligencias para mejor proveer de José Martín Ostos, Catedrático de Derecho Procesal en la Universidad de Sevilla; ${ }^{91}$ o la figura del secretario judicial en dicho procedimiento de Manuel Domínguez Moreno. ${ }^{92} \mathrm{Y}$ otros son más generales, cual es el caso del estudio sobre la interrelación entre el procedimiento gubernativo y el proceso civil de Julio García Casas, Profesor Titular de Derecho Procesal de la Universidad de Sevilla y que tiene una gran proyección social en el mundo de la cultura musical clásica, desempeñando actualmente la Presidencia Nacional de Juventudes Musicales; ${ }^{93}$ y del recurso de nulidad en el proceso civil de Manuel Morón Palomino, Catedrático de Derecho Procesal de la Universidad de La Laguna. ${ }^{94}$

No faltan tampoco estudios sobre procesos civiles especiales, como es el caso de la tesis con acentuado perfil histórico que tiene como objeto el juicio ejecutivo del Magistrado del Tribunal Superior de Andalucía Vidal Estepa Moriana, ${ }^{95} \mathrm{o}$ la que trata de los interdictos con un planteamiento reformista de Manuel González Aguilar. ${ }^{96}$

Otras jurisdicciones también han sido objeto de estudios. Así la jurisdicción penal sobre un aspecto particular, concretamente el secreto en la prueba de testigos en el proceso penal de Víctor Manuel Moreno Catena, Ex-Decano de la Facultad de Derecho de Sevilla y actualmente Catedrático de Derecho Procesal de la Universidad Carlos III; ${ }^{97}$ en tanto que la jurisdicción militar ha sido investigada con vistas a una presentación general sobre sus fundamentos y extensión por Manuel Gómez del Castillo, Profesor Titular de Derecho Procesal de la Universidad de Huelva. ${ }^{98}$

José Almagro Noste, La prueba de informes en el proceso civil, 1966; Director: Faustino Gutiérrez-Alviz Armario; FDEB TD-033.

91. José de los Santos Martín Ostos, Para mejor prover en el proceso civil español actual (estudio doctrinal, legislativo y jurisprudencial), 1976; Director: Faustino Gutiérrez-Alviz; FDEB TD 094.

92. Manuel Domínguez Moreno, El secretario judicial en el proceso civil español, 1976; Director: Faustino GutiérrezAlviz; FDEB TD-097.

93. Julio García Casas, Via gubernativa y proceso civil, 1974; Director: Faustino Gutiérrez-Alviz Armario; FDEB TD-076.

94. Manuel Morón Palomino, La nulidad en el proceso civil español, 1957; Director: Faustino Gutiérrez-Alviz Armario; FDEB TD-003.

95. Vidal Estepa Moriana, Nuevas orientaciones sobre la historia del juicio ejecutivo en España, 1976; Director: Faustino Gutiérrez-Alviz; FDEB TD-089.

96. Manuel González Aguilar, Hacia una reforma de los interdictos, 1962; Director: Faustino Gutiérrez-Alviz Armario; FDEB TD-022.

97. Víctor Manuel Moreno Catena, El secreto en la prueba de testigos en el proceso penal, 1979; Director: Faustino Gutiérrez-Alviz Armario; FDEB TD-109.

98. Manuel Gómez del Castillo y Gómez, El fundamento y la extensión de la jurisdicción militar, 1972; Director Faustino Gutiérrez-Alviz Armario; FDEB TD-059. 
3. En un tercer grupo, se han defendido entre cinco y diez tesis doctorales, en las áreas de Derecho Internacional, Filosofia del Derecho, Derecho Constitucional y Derecho Romano.

Derecho Internacional. Dentro de esta primera etapa encontramos un grupo de tesis doctorales que fueron dirigidas en su mayoría por los Catedráticos de Derecho Internacional Mariano Aguilar Navarro y su sucesor en la Cátedra Francisco Sánchez Apellániz. Un número importante de ellas tienen como contenido determinados problemas técnico-jurídicos de la comunidad europea en sus sucesivos momentos evolutivos. Así las tesis doctorales dedicadas a las fuentes del derecho internacional, como son los casos de la realizada por José Antonio Pérez Beviá, Catedrático de Derecho Internacional en la Universidad de Córdoba, sobre el reglamento en las comunidades europeas; ${ }^{99}$ o la llevada a cabo por Lucía Millán Moro, Catedrática de Derecho Internacional en la Universidad Pablo de Olavide, sobre la armonización de las legislaciones en la Comunidad económica europea. ${ }^{100}$

En este grupo podemos incluir también la primera que se leyó en este campo del saber jurídico, realizada por Juan Antonio Carrillo Salcedo, actual Catedrático de Derecho Internacional Público en la Universidad de Sevilla y juez del Tribunal de Estrasburgo durante un número cuantioso de años, sobre la recepción del recurso contencioso administrativo en el sistema jurídico de la Comunidad económica del carbón y del acero; ${ }^{101} \mathrm{o}$ la de José Manuel Peláez Marón, también Catedrático de Internacional Público en la Facultad de Derecho de Sevilla y titular de la Cátedra Jean Monet de la Comunidad Europea, quien investigó sobre el recurso de infracción en el Tratado de la Comunidad Económica Europea. ${ }^{102}$ De otra parte la asociación con la comunidad económica europea es el asunto de la tesis doctoral de Alejandro Vázquez Labourdette. ${ }^{103}$

A determinados aspectos de las organizaciones internacionales están dedicadas dos tesis doctorales: de Julio D. González Campos, actual Catedrático de Derecho Internacional de la Universidad Complutense, sobre la admisión de estados en dichas organizaciones inter-

99. José Antonio Pérez Beviá, El Reglamento como fuente de derecho en las comunidades europeas, 1971; Director: Francisco Sánchez-Apellániz Valderrama; FDEB TD-055. 100. Lucia Millán Moro, La armonización de legislaciones en la CEE, 1982; Director: Francisco Sánchez-Apellániz 1.derrama; FDEB TD-122.

. Juan Antonio Carrillo Salcedo, La recepción del recurso contencioso administrativo en el sistema jurídico de la comunidad europea del carbón y del acero, 1958; Director: Mariano Aguilar Navarro; FDEB TD-9.

02. José Manuel Peláez Marón, El recurso de interpretación en el tratado de la Comunidad Económica Europea, 1968 irector: Francisco Sánchez-Apellániz Valderrama; FDEB TD-038. 103. Alejandro Vázquez Labourdette, La asociación con la Comunidad Económica Europea, 1969; Director: Francisco nacionales; ${ }^{104}$ y la de José A. Girón Larrucea, Profesor Titular de Derecho Internacional en la Universidad de Sevilla, sobre el fundamento y caracteres esenciales de los actos unilaterales de las organizaciones internacionales. ${ }^{105} \mathrm{El}$ estudio de la Guerra Civil y el Derecho Internacional constituye el objeto de la tesis doctoral de Roberto Mesa Garrido, Catedrático de Derecho Internacional de la Universidad Complutense de Madrid; ${ }^{106}$ y la ambigua situación jurídico-pública de Puerto Rico es el tema de la del Dr. Israel Molina Robles. ${ }^{107}$

Filosofia del Derecho. Durante esta primera etapa las tesis doctorales que se leen tienen como director casi exclusivo al Prof. Elías de Tejada y el tema suele ser el pensamiento jurídico político y social de determinados autores clásicos como el Padre P. Ramírez de Vicente Marrero Suárez; ${ }^{108}$ el de James Harrington de Pablo Badillo O’Farrell, actual Catedrático de Filosofia del Derecho de la Universidad de Sevilla; ${ }^{109}$ el de Francisco Núñez de Pineda y Bascuñán de Ramón Soriano Díaz, actual Catedrático de Filosofia del Derecho en la Universidad de Huelva, ${ }^{110}$ y Francisco de Quevedo de Antonio Ruiz de la Cuesta, Profesor Titular de Filosofia del Derecho en la Facultad de Derecho de Sevilla, ${ }^{111}$ o más actuales como Pedro Antonio de Alarcón de Manuel Porras y del Corral ${ }^{112}$ y Giorgio del Vecchio de José Miguel Pavón Ruiz. ${ }^{113}$

Derecho Financiero. Todas las tesis doctorales de esta primera época han sido dirigidas por el Catedrático de Economía Política, Hacienda Pública y Derecho Financiero, Prof Dr. Jaime García Añoveros, quien desempeñó el cargo de Ministro de Hacienda en el Gobierno de la Unión de Centro Democrático, durante la Transición. El número de

104. Julio D. González Campos, La admisión de Estados en las organizaciones internacionales, 1960; Director: Marian Aguilar Navarro; FDEB TD-017.

05. José A. Girón Larrucea, Fundementos $y$ corncteres esenciales de los atos unibterales de las organizaciones internacionales, 1980; Director: Francisco Sánchez-Apellániz; FDEB TD-112.

106. Roberto Mesa Garrido, La Guerra civil y el Derecho Internacional, 1962; Director: Francisco Sánchez-Apellániz; FDEB TD-20.

107. Israel Molina Robles, Puerto Rico: su lucha por un status, 1977; Director: Francisco Sánchez-Apellániz Valderama; FDEB TD-100.

108. Vicente Marrero Suárez, La fundamentación filosófica de una filosofia jurídico-social en la obra del P. Ramírez, 1970; Director: Francisco Elías de Tejada; FDEB TD-051.

109. Pablo J. Badillo O'Farrell, La filosofía politico-juridica de James Harrington, 1972; Director: Francisco Elías de Tejada; FDEB TD-062.

Tamón Soriano Díaz, El pensamiento jurídico de Francisco Núñez de Bascuñán, 1976; Director: Francisco Elías de Tejada; FDEB TD-058.

11. Antonio Ruiz de la Cuesta, Filosofia jurídico-politica de Don Francisco de Quevedo, 1981; Director: Antonio

12. Manuel Porras y del Corral, Ideas politicas y sociales en Pedro A. de Alarcón, 1971; Director: Francisco Elías de Tejada; FDEB TD-054

(1965: Director: Francisco Flís de Tejada: FDEB TD-028. 
doctores que constituyen sus discípulos es mayor que el que aquí se refleja, pues en este área, al igual que sucede en la de Derecho Mercantil, algunos doctorandos colacionaron su grado de doctor en la Universidad de Bolonia. Los temas de las tesis presentan un panorama muy disperso, como consecuencia de la integración tripartita que en un principio tuvo este saber jurídico. Así encontramos una que es más propia de la Economía Política y que tiene por objeto el estudio socioeconómico de la humedografía de Galicia de José Manuel Beiras Torrado; ${ }^{114}$ otra que es más encuadrable en el campo de la Hacienda Pública y que tiene un marcado perfil histórico-jurídico y se refiere a los origenes del Derecho presupuestario en España de Esteban López-Escobar, actual Catedrático y Decano de la Facultad de Ciencias de la Información de la Universidad de Navarra. ${ }^{115}$ Más propiamente financieras son las que se refieren a la sustitución financiera de Emilio Pérez Ruiz, Profesor Titular de Derecho Financiero de la Universidad de Sevilla; ${ }^{116}$ los jurados tributarios de María Antonia Víbora Rodríguez, que fue la segunda mujer doctora de la Facultad de Derecho de Sevilla; ${ }^{117}$ y los aspectos jurídico-tributarios de la concentración de empresas de Rafael Navas Vázquez, Catedrático de Derecho Financiero de la Universidad de Huelva y que desempeña actualmente el cargo de Presidente de la Cámara de Cuentas de la Comunidad Autónoma de Andalucía. ${ }^{118}$

Derecho Político/Constitucional. La primera de las tesis leída tiene un marcado carácter constitucionalista, aunque lógicamente no referida a la realidad hispánica; pero ciertamente constituye un detalle de valentía científica si se tiene en cuenta que se elaboró y defendió en el segundo lustro de los años cincuenta, en plena efervescencia de la consolidación de un régimen tan poco constitucional como el franquista: se trata de un trabajo de investigación que tuvo como objeto la Constitución británica ${ }^{119}$ y cuyo autor fue el Profesor Adjunto de Derecho Político Dr. Manuel Romero Gómez, quien dejó una honda huella en la formación jurídica democrática de su alumnado, entre cuyos miembros me encuentro. Otros temas, aunque españoles, tienen un perfil histórico jurídico muy marcado, circunstancia muy común en el campo del Derecho Político durante la Dictadura de Franco, hasta el punto de que, como es sabido, gran parte de la Historia del Derecho Constitucional es obra de ellos. Así el estudio sobre la opinión pública espa-

114. Jose

114. José Manuel Beiras Torrado, Contribución al estudio socioeconómico de la demografía de Galicia, 1965; Director Jaime García Añoveros / Jesús Prados Arrarte; FDEB TD-027

115. Esteban López-Escobar Fernández, Los origenes del derecho presupuestario en España, 1967; Director: Jaime García Añoveros; FDEB TD-036.

116. Emilio Pérez Ruiz, La sustitución tributaria, 1969; Director: Jaime García Añoveros; FDEB TD-045.

117. $\mathrm{M}^{2}$ Antonia Víbora Rodríguez, Los jurados tributarios, 1972; Director: Jaime García Añoveros; FDEB TD-065. 118. Rafael Navas Vázquez, Aspectos jurídico-tributarios de la concentración de empresas, 1975; Director: Jaime García veros; FDEB TD-084.

119. Manuel Romero Gómez, La Constitución británica, 1958; Director: Ignacio Lojendio e Irure; FDEB TD-010. ñola y las Cortes de Cádiz frente a la emancipación hispanoamericana de Eduardo Pérez Guilhou; ${ }^{120}$ o el que se refiere a las elecciones en Sevilla y su provincia durante la Segunda República de José Gómez Salvago; ${ }^{121}$ e igual podemos decir de la tesis sobre el pensamiento político de P. J. Proudhon de Antonio Porras Nadales, actual Catedrático de Derecho Constitucional en la Universidad de Huelva, ${ }^{122}$ seguramente elaborada en pleno período constituyente. Otras se presentan con un planteamiento más técnico-jurídico, como son los casos de las dedicadas a la reserva de ley de Javier Pérez Royo, Catedrático de Derecho Constitucional de la Universidad de Sevilla, de la que ha sido Rector Magnífico; ${ }^{123}$ o la que tiene por objeto el estado de sitio como forma de protección extraordinaria del Estado del actual Presidente del Tribunal Constitucional Pedro Cruz Villalón. ${ }^{124}$ Todas ellas tienen de común el haber sido dirigidas con anterioridad a la promulgación de la Constitución de 1978 por el Prof. Lojendio e Irure, Catedrático de Derecho Político.

Derecho Romano. Determinados aspectos del derecho público, del derecho matrimonial y del derecho de sociedades en su perfil procesal han sido los tres temas tratados por los doctores de esta área jurídica durante esta primera etapa. En el Derecho Público podemos encuadrar dos estudios, uno sobre la soberanía en Roma de José Olivares d'Angelo que terminaría desempeñando su actividad docente como Profesor Titular de Derecho Internacional Privado en la Universidad de Sevilla; ${ }^{125}$ y otro sobre la libertas de David García Leo, quien durante muchos años desempeñaría la docencia del Derecho Romano en calidad de Profesor encargado de curso. ${ }^{126}$ En cuanto al derecho matrimonial se han estudiado los aspectos referidos al origen y fundamento de la prohibición de donaciones inter virum et uxorem por Ramón López Rosa, actual Catedrático de Derecho Romano en la Universidad de Huelva en cuya Facultad de Ciencias Jurídicas y Sociales es Decano; ${ }^{127}$ y el divorcio en las leyes augústeas por Concepción

120. Eduardo Pérez Guilhou, La opinión pública española y las Cortes de Cádiz frente a la emancipación hispanoamericana, 1808-1814 (aportación de datos para su estudio), 1960; Director: Ignacio de Lojendio e Irure; FDEB TD-015. 121. José Gómez Salvago, La Segunda República: elecciones en Sevilla y su provincia, 1977; Director: Ignacio María Lojendio Irur; FDEB TD-098.

122. Antonio Porras Nadales, El pensamiento politico de P.-J. Proudhon, 1978; Director: Ignacio María Lojendio Iru122. Antonio Por
re; FDEB TD-103.

123. Francisco Javier Pérez Royo, La reserva de Ley, 1969; Director: Ignacio Lojendio e Irure; FDEB TD-046. 124. Pedro Cruz Villalón, El estado de sitio como forma de protección extraordinaria del Estado, 1975; Director: Ignacio María Lojendio Irure; FDEB TD-085.

125. José J. Olivares d'Angelo, La soberanía en Roma, 1960; Director: Dr. F. Pelsmaeker e Iváñez FDEB TD-014. 126. David García Leo, La "libertas" en Roma: principalmente a través de Cicerón, 1978; Director: J. Aparici Díaz; 126. David García Leo, La "libertas" en Roma: principalmente a través de Ciceron, 1978; Director: J. Aparici Diaz;
FDEB TD-105. DEB TD-105.

127. Ramón López Rosa, Origen y fundamento de la prohibición de las donaciones inter virum et uxorem, 1976; Director: José Aparici Díaz; FDEB TD-093. 
Gómez Ruiz. ${ }^{128} \mathrm{Y}$ por lo que se refiere al derecho de sociedades la tesis sobre la fórmula de la actio pro socio realizada por Carmen Velasco García. ${ }^{129}$ Ambas doctoras desempeñan actualmente la docencia del Derecho Romano con la cualificación de Profesoras Titulares; la primera en la Universidad de Sevilla y la segunda en la recién creada Universidad Pablo de Olavide, donde ejerce el cargo de Decana de la Facultad de Ciencias Jurídicas y Sociales.

4. Por fin, en un último grupo, se encuentran las áreas de conocimiento con menos de cinco tesis doctorales defendidas, en Derecho Mercantil y Canónico.

Derecho Mercantil. Llama la atención el bajo número de tesis doctorales que se defienden en esta primera etapa en este área de conocimiento; esta circunstancia se debe a que los doctorandos de la misma solían y suelen realizar sus estudios de doctorado en la Universidad de Bolonia como becarios del Colegio de San Clemente. En todo caso, entre 1955 y 1985 encontramos tres Doctores iuris de la Real Fábrica de Tabacos, cuyas tesis doctorales fueron realizadas bajo la dirección del Catedrático de Derecho Mercantil y Doctor por Bolonia Manuel Olivencia Ruiz. Concretamente son: la que está dedicada al marco jurídico de las cooperativas agrarias españolas de comercialización de Rafael Carbonell de Many; ${ }^{130}$ la que tiene como tema los aspectos jurídicos de las operaciones de rentas con primas de Juan Font Galán, actual Catedrático de Derecho Mercantil de la Universidad de Córdoba; ${ }^{131}$ y la que estudia la obligación de intereses en el Derecho Mercantil español de David Morán Bovio, Catedrático de Derecho Marítimo de la Universidad de Cádiz. ${ }^{132}$

Derecho Canónico. En esta primera etapa sólo se leyeron tres tesis doctorales, de las cuales dos tienen un perfil histórico jurídico muy marcado. Así la de Carlos Seco Caro, Profesor Titular de Derecho Eclesiástico del Estado de la Universidad de Sevilla es de tema indianista, trata de los privilegios martrimoniales indianos; ${ }^{133}$ y la de Juan José Rubio Rodríguez sobre las causas pías de los juristas clásicos españoles en los siglos XV a

128. Concepción Gómez Ruiz, El divorcio y las leyes augústeas, 1985; Director: José Aparici Díaz; FDEB TD-133. 129. Carmen Velasco García, La fórmula de la actio pro socio, 1984; Director: José Luis Murga Gener / José Aparici 129. Carmen Velasco García, La formula de la aro pro 130. Rafael Carbonell de Many, El marco juŕdico de las cooperativas agrarias españolas de comercialización: revisión y
nueva perspectiva, 1971; Director: Manuel Olivencia Ruiz; FDEB TD-053.

131. Juan Ignacio Font Galán, Aspectos juridicos de las operaciones de ventas con primas, 1974; Director: Manuel Olivencia Ruiz; FDEB TD-075

132. David Morán Bovio, La obligación de intereses en el derecho mercantil español, 1985; Director: Manuel Olivenci Ruiz; FDEB TD-132.

133. Carlos Seco Caro, Los privilegios matrimoniales indianos (estudio sistemático-funcional), 1958; Director: Manue Giménez Fernández; FDEB TD-011
XVII. ${ }^{134}$ La tercera sobre el derecho vigente se debe a Jerónimo Borrero Arias, actualmente Profesor Titular de Derecho Canónico en la Universidad Plablo de Olavide, tiene como contenido un estudio comparativo de la adopción entre el derecho civil y el derecho canónico. ${ }^{135}$

RETAZOS DE LA PROYECCIÓN ACADÉMICA, SOCIAL Y POLÍTICA DE LOS DOCTORES IURIS DE LA

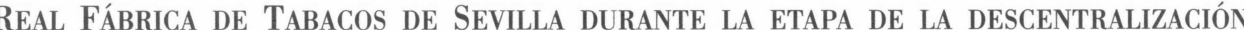
(1955-1985)

De los ciento treinta y siete doctores en Derecho de la Real Fábrica de Tabacos de Sevilla a quienes se colacionó el grado académico de Doctor durante la etapa de la descentralización (1955-1999), cincuenta y uno alcanzaron la categoría profesoral de Catédráticos de Universidad y 24 la de Profesor Titular de Universidad. Es decir más de la mitad, o sea 75, han obtenido una importante promoción académica.

En este sentido la más sobresaliente es el área de Derecho del Trabajo, de cuyos veinte doctores, diez son Catedráticos de Universidad y cinco Profesores Titulares. Pero además este área sobresale por la proyección político social de sus Doctores: pues el Dr. Rodríguez Piñero, Catedrático de Universidad, que desempeñó el cargo de Decano de la Facultad durante la Transición, ha ocupado el cargo de Magistrado del Tribunal Constitucional y además ha llegado a ser su Presidente. De otra parte el Doctor José Rodríguez de la Borbolla ha desempeñado la Presidencia de la Junta de Andalucía y el Dr. Manuel Chaves, que ha sido Ministro de Trabajo, actualmente es el Presidente de la Comunidad Autónoma de Andalucía. A ambos les dirigió la tesis el Dr. Rodríguez Piñero y son Profesores Titulares de Derecho del Trabajo.

En el área de Derecho Internacional debemos destacar al Dr. Carrillo Salcedo que ha sido miembro del Tribunal Europeo de Derechos Humanos con sede en Estrasburgo; y al Dr. González Campos, miembro actual del Tribunal Constitucional. En Derecho Constitucional debemos traer a colación al Dr. Javier Pérez Royo, que ha sido Rector de la Universidad hispalense; y al Dr. Jesús Cruz Villalón que es actualmente Presidente del Tribunal Constitucional. Por lo que se refiere al área de Derecho Administrativo debemos citar a la Doctora Amparo Rubiales, que ha tenido una fuerte proyección

134. Juan José Rubio Rodríguez, Las causas pias en los juristas clasicicos españoles de los siglos XV al XVII (estudio histórico-jurídico), 1976; Director: Alberto Bernárdez Cantón; FDEB TD-091.

35. Jeronimo Borrero Arias, Conexiones en materia de adopción entre el derecho canonico y el derecho civil, 1976; Director: Alberto Bernárdez Cantón; FDEB TD-095. 
política como Diputada en los Parlamentos autonómico y nacional, Senadora y Delegada del Gobierno en Andalucía. En cuanto al área de Derecho Financiero, el Dr. Rafael Navas desempeña actualmente la Presidencia de la Cámara de Cuentas de Andalucía.Y finalmente en el área de Derecho Civil nos encontramos con la Dra. Rosario Valpuesta, que desempeña el cargo de Rectora en la Universidad Pablo de Olavide; el Dr. Ángel López López, que fue Consejero de Gobernación de la Junta de Andalucía y Presidente del Parlamento Andaluz; y el Dr. José Antonio Infantes Florido, primer Doctor de la época que tratamos y que accedió al orden episcopal, y fue Obispo de la Diócesis de Córdoba durante los años setenta. 